\title{
A juiced-up joint implant
}

With a slight touch of chemistry, polyethylene, the common plastic used in grocery bags and packaging materials, becomes Ultra-High-Molecular-Weight Polyethylene (UHMWPE), a long-chain and super strong cousin better suited for medical applications than for carrying home takeout.

UHMWPE has been used for decades as a biomaterial for joint replacements, but until the late 1990s, even UHMWPE was no match for the daily grind encountered by most weight-bearing joints. So called 'wear particles' would often break free from the implant, causing accumulation in the joint and a destructive process known as osteolysis to the surrounding bone, eventually leading to implant failure.

In 1998, researchers in the Harris Orthopedics Laboratory at the Massachusetts General Hospital developed a highly crosslinked version of UHMWPE that resists breakdown and wear particles, an improvement with such clinical benefits that it rapidly became the standard of care for total hip replacements, eliminating $\sim 90 \%$ of osteolytic UHMWPE implant failures.

But wear-and-tear induced osteolysis is not the only source of UHMWPE implant problems; bacterial infection during and after surgery can also lead to implant failure. Although relatively rare, when a joint implant does get infected, it's serious bad news for the patient. According to Dr. Ebru Oral, associate director of biomaterials in the Harris Orthopedics Lab, "although infections still only affect a small number of overall patients, probably about $1 \%$, it's quite a morbid problem and the patient's quality of life decreases a lot."

In her 15-year tenure in the Harris Lab, first as a postdoc and now independent researcher, she has constantly worked to improve the performance of UHMWPEimplants. Now, with an eye towards solving implant infections, her team in the Harris Lab has created a novel drug-eluting joint implant that they hope will help improve recovery in patients suffering from infections, and potentially eliminate infections from happening in the first place (Nat. Biomed. Eng. 1, 0080; 2017).

When a UHMWPE implant gets infected, the current standard of treatment requires patients to go through a two stage process. First, the implant is removed and a dummy implant (or 'spacer') made of antibioticeluting bone cement is put in its place for months, helping to kill off the bacteria. A second surgery is then done to remove the bone cement spacer and a fresh prosthetic put back into place.

This standard, although quite effective, is hardly ideal. In addition to requiring multiple invasive surgeries, bone cement is not mechanically stable enough to bear weight, so patients are on crutches for the duration of phase- 1 while they wait for bacteria to be cleared. The novel UHMWPE implant developed by Oral's team can itself elute antibiotics, potentially eliminating the need for a bone cement dummy implant and trimming the treatment strategy down to a single surgery.

While adding in drug-elution capabilities into a weight-bearing UHMWPE implant might seem like an obvious way to bypass the bone cement middleman in current standard of care practices, the engineering behind it is far from simple. Both UHMWPE and bone cement are polymers, and additional components, like antibiotics, have to be mixed in. The more antibiotic mixed into the polymer, the more that will elute into the joint to kill off bacteria, but there's a steep tradeoff: more antibiotics in the mix also means the polymer becomes more fragile and less capable of bearing weight.

How the antibiotics mix into the material also impacts how efficiently they will elute. Antibiotics mixed into bone cement, for example, form inefficient spherical clusters, with only a small amount actually escaping into the joint. To reach a sufficient concentration to kill off bacteria, bone cement requires a lot of antibiotics mixed into its polymer, making it less capable of bearing weight (and hence the crutches for the patient).

As Oral's team describes in their paper, antibiotics added into UHMWPE polymers form eccentric and highly interconnected drug clusters, which elute with significantly greater efficiency than spherical clusters in bone cement. Therefore, less drug is required in the polymer mix to reach critical concentrations in the joint and the UHMWPE can maintain its mechanical strength and weight-bearing properties, functioning as a replacement implant while also fighting off the infection. In addition to several in vitro assays to quantify drug elution properties, the team tested their new implant in vivo, using New Zealand White rabbits with localized infections of $S$. aureus in the tibia, finding that the antibiotic-eluting UHMWPE implant was better at eliminating infections, both from free floating bacteria and bacteria in biofilms, than bone cement.

Oral describes the Harris Lab as an "umbrella laboratory" that mixes physicians, scientists and engineers, all within the clinical setting of Mass General Hospital. "We're a translational laboratory, so we are really trying to focus our science on practical concerns for the patient." With plenty of potential applications on the horizon for their drug-eluting implant, Oral wants to focus first on using it to treat infections (as opposed to prophylaxis, which is currently not on option in the US, and could require years to get regulatory approval). "There's a lot that we can do with this material, but if we can't put it into the patients, then there's no point in developing this." Because their implant uses materials-including the antibiotics - that are all separately approved and in widespread clinical use, she hopes they can be put to the test in humans soon.

Dustin M. Graham 